\title{
Mercaptohexanol assembled on gold: FM-AFM imaging in water
}

\author{
Takumi Hiasa, Hiroshi Onishi* \\ Department of Chemistry, School of Science, Kobe University, Rokko-dai, Nada, Kobe, \\ 657-8501 Japan \\ ${ }^{*}$ Corresponding author. E-mail address: oni@kobe-u.ac.jp
}

\begin{abstract}
Mercaptohexanol is adsorbed on gold (111) and probed in an aqueous $\mathrm{KCl}$ solution by frequency-modulation atomic force microscopy (FM-AFM). Two different features, dot-like and rod-like protrusions, appear in the surface topography. Laterally periodic modulation of the solution density is recognized in the tip-surface force distribution cross-sectional to the surface. A possible assembly of mercaptohexanol, in which two mercaptohexanol molecules are laid down in a rectangular $(3 \times 2 \sqrt{ } 3)$ unit cell, is proposed to explain the experimental results.
\end{abstract}

\section{Keywords}

Self-assembly; Atomic force microscopy; Interfacial water; Hydrophilic property; Thiols

(C) 2013. This manuscript version is made available under the Elsevier user license http://www.elsevier.com/open-access/userlicense/1.0/ 


\section{Introduction}

Self-assembled monolayers have attracted attention over the years due to scientific interest in molecular systems and the engineering aspects of functionalized surfaces [1,2]. Alkanethiols on gold provide a class of well-controlled organic monolayers. Some thiols, particularly when functionalized with hydrophilic end-groups, are dissolved in water and deposited from the aqueous solution onto the substrate. It is thus important to know how the structures of monolayers are assembled and preserved in water. Thiols with long alkyl chains prefer a densely packed assembly with their molecular axis upright to the surface. The assembly of $\mathrm{C}_{3}$ to $\mathrm{C}_{6}$ thiols is still a controversial issue, however, although low-coverage phases have been reported and ascribed to a reduced preference for upright chain stacking [3-10].

In this study, the structure of a mercaptohexanol $\left(\mathrm{HSC}_{6} \mathrm{H}_{11} \mathrm{OH}\right)$ monolayer on gold (111) is investigated in an aqueous $\mathrm{KCl}$ solution. The symmetry of a long-range ordered monolayer of this compound has been identified to be either $(3 \times \sqrt{ } 13)$ [9] or $(3 \times 2 \sqrt{3})$ [10] in previous studies using scanning tunneling microscopy (STM). However, the placement of adsorbed molecules has not yet been revealed in unit cells. Here, frequency-modulation atomic force microscopy (FM-AFM) is used to determine the placement of the functionalized thiol. Microscopes equipped with low-noise optical deflection sensors [11] are able to trace the atomic-scale topography of a number of solid objects immersed in water, including mica [12], calcite [13], lysozyme [14], lipid bilayers [15], and DNA [16].

One further advantage of current FM-AFM technology is the ability to visualize the liquid structure at interfaces. Liquid density is no longer uniform when structured at an interface. The tip-surface force is perturbed where the liquid is locally dense or dilute, as demonstrated in water on mica [17, 18], sapphire [19], OH- or $\mathrm{COOH}$-terminated thiolate monolayer [20], lipid bilayers [21], etc. Visualization in organic solvents (dodecane, hexadecane and phenyloctane) is also possible [22]. A recent study of water on crystalline p-nitroaniline by the authors [23] showed that water density was modulated on the amino and nitro end-groups exposed on the surface, not on the benzene ring. Mercaptohexanol contains $\mathrm{OH}$ and $\mathrm{SH}$ end-groups with an $n$-hexyl chain in between. The structure of water exposed to the mercaptohexanol monolayer, if observable, should provide knowledge about where the end-groups are placed in the unit cell.

\section{Experimental}

The monolayer of mercaptohexanol was prepared on gold film. Cleaved mica wafers were annealed at $450{ }^{\circ} \mathrm{C}$ in a vacuum of $10^{-5} \mathrm{~Pa}$ and exposed to a gold vapor source, in a 
custom-designed chamber (Organoworks). The film thickness was $150 \mathrm{~nm}$. The gold-deposited wafers were immersed overnight in an aqueous solution of mercaptohexanol (Aldrich, 97\%) with purified water (Millipore) of $10 \mu \mathrm{mol} \mathrm{l}^{-1}$.

A Shimadzu SPM 9600 microscope was modified using the same method used by Fukuma et al. [11] to reduce the noise of the cantilever deflection to as low as $20 \mathrm{fm} \mathrm{Hz}^{-1 / 2}$. Silicon cantilevers ( $\mathrm{NCH}$, Nanosensors) with a nominal spring constant of $40 \mathrm{~N} \mathrm{~m}^{-1}$ were backside-coated with aluminum or gold. The resonant frequency of the cantilever was 130-150 $\mathrm{kHz}$ and the quality factor $(Q)$ of resonance was 5 in the imaging liquid, an aqueous $\mathrm{KCl}$ solution of $0.05 \mathrm{~mol}^{-1}$. The solution was prepared with $\mathrm{KCl}$ (Nakarai, 99.5\%). The electrolyte was required to reduce the decay length of the electric double layer. The estimated Debye length was $0.9 \mathrm{~nm}$ in the presence of the $\mathrm{KCl}$ solute. A droplet of the imaging liquid was placed on the wafer and then the cantilever assembly was placed on the top. The liquid droplet on the wafer was open to the environment. Imaging scans were performed at room temperature.

To stabilize a self-oscillation loop in the low- $Q$ environments, bandpass filters $(Q=20)$ were inserted into the loop. The absolute deflection of the cantilevers was calibrated using the theoretical amplitude of the thermally induced Brownian motion.

\section{Results and discussion}

\subsection{Topography of the mercaptohexanol monolayer}

The topography of the mercaptohexanol-adsorbed gold film was determined in the $\mathrm{KCl}$ solution. To trace the topography, the tip-surface distance was regulated to keep the resonance frequency shift of cantilever oscillation $(\Delta f)$ constant. In repeated imaging scans, two topographic features were recognized with different frequencies. The size of the rectangular unit cell was consistent in the two appearances, $0.8-0.9 \mathrm{~nm}$ along the short axis and $1.0 \mathrm{~nm}$ along the long axis. The lengths reproduced those of the $(3 \times 2 \sqrt{ } 3)$ lattice: $0.87 \mathrm{~nm} \times 1.0 \mathrm{~nm}$, reported on thiol monolayers [24, 25].

The dot-like features appearing frequently are shown in Fig. 1 (a). The $(3 \times 2 \sqrt{3})$ rectangular lattice is equally described as a $c(4 \times 2)$ superlattice of a $(\sqrt{3} \times \sqrt{3}) \mathrm{R} 30^{\circ}$ lattice relative to the hexagonal unit cell of gold (111). One unit cell of the $(3 \times 2 \sqrt{3})$ lattice contains four $(\sqrt{3} \times \sqrt{3})$ cells. Four dot-like protrusions were recognized in a $(3 \times 2 \sqrt{3})$ cell with different levels of brightness. The protrusions at the cell corners were brightest. Two were inside the cell with moderate brightness. The last ones, at the centers of the long edges, cannot be acknowledged, i.e., they appear with low brightness. 
The $(3 \times 2 \sqrt{3})$ cell containing protrusions of different brightness levels was observed in STM studies of vapor-deposited [9] and solution-deposited [10] mercaptohexanol monolayers on gold. Interpretation of these protrusions is not straightforward. Different conformations of the adsorbed thiolate [6] and different sites of thiolate adsorption on gold [26] have been proposed.

Figure 1(b) shows the other features appearing less frequently. Rod-like features, unlike the dot-like protrusions in (a), appeared on the surface, while the shape and size of the unit cell remained intact. The length of each rod was 1.3-1.6 nm. Since the extended length of a mercaptohexanol molecule is $0.8 \mathrm{~nm}$, the rod was assigned to a pair of two mercaptohexanol molecules laid on the surface. A possible structure for the lying adsorbates is illustrated in Figure 1(c). In drawing the picture, the $\mathrm{OH}$ and $\mathrm{SH}$ end-groups are assumed to occupy the threefold-hollow sites on the $(\sqrt{3} \times \sqrt{3})$ lattice, as suggested in a previous study [27].

The structure proposed in (c) contains two mercaptohexanol molecules in a $(3 \times 2 \sqrt{3})$ cell. There is enough space available to place four lying molecules in the cell, when they are closely packed on the surface [9]. Is it possible that two more molecules were present in the cell? It is difficult to find reasons for the two mercaptohexanol molecules to be visible and the other two to be invisible to the probe. Imaging scans to produce the topography seen in Figure 1 were performed in the repulsive tip-surface force regime, as evidenced by positive $\Delta f$ setpoints. The repulsive force is caused by the exclusion principle and hence is to be expected on a molecular substance of any conformation. Therefore, a structure with two adsorbed mercaptohexanol molecules per $(3 \times 2 \sqrt{ } 3)$ cell is proposed.

Vacant volume is available for additional adsorption in the proposed $(3 \times 2 \sqrt{3})$ structure. An attempt was made to fill this vacant volume with additional mercaptohexanol. The monolayer shown in Figure 1 was prepared by immersion for 10 minutes in the solution of $10 \mu \mathrm{mol} \mathrm{l}^{-1}$. Immersion for 40 minutes in a solution of $100 \mu \mathrm{mol} \mathrm{l}^{-1}$ was employed in a previous STM study [10]. However, immersion in the solution of $10 \mu \mathrm{mol} 1^{-1}$ for a longer time resulted in nanometer-sized aggregates on the surface. Molecular-scale imaging was not achieved on the surface with the aggregates. 
(a)

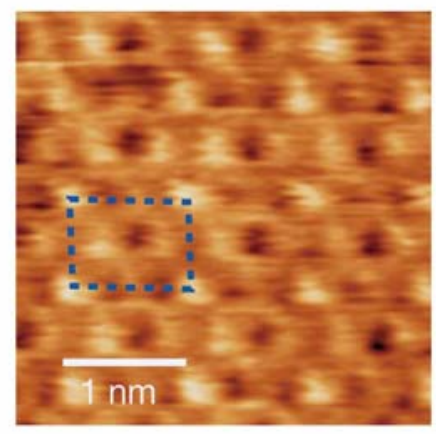

(b)

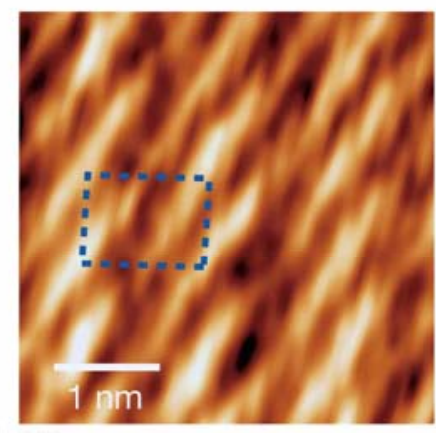

(c)

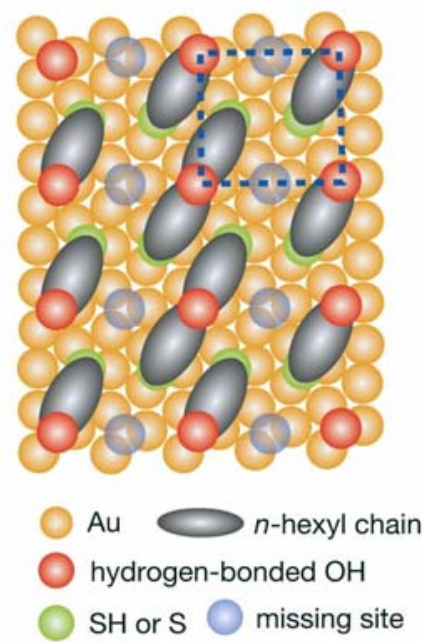

Fig. 1. Topography of the mercaptohexanol monolayer on gold observed in a $\mathrm{KCl}$ solution. The (a) dot-like and (b) rod-like features. The cantilever oscillation amplitude is $0.1 \mathrm{~nm}$ and the frequency shift setpoint is (a) $+700 \mathrm{~Hz}$ and (b) $+400 \mathrm{~Hz}$. (c) The structure of the mercaptohexanol monolayer proposed in this study. 
Transition from the dot-like features to the rod-like features and vice versa occurred even in one imaging scan. Figure 2 shows the topography obtained in one scan with two transitions. The tip was scanned from left to right. A lateral scan was completed in $80 \mathrm{~ms}$, and then the tip was shifted down to start another scan. Rod-like features initially appeared and then suddenly changed to dot-like features on the scan line marked with the first arrow. A reverse change from dots to rods happened on the line with the second arrow. The raw topographic image is shown in the main figure without collecting the steady drift of the object in the vertical direction. The three insets present drift-collected images to identify the rod-like, dot-like, and again rod-like features that appeared in the topography. The instantaneous and reversible transitions from one appearance to the next happened despite the constant cantilever oscillation amplitude and $\Delta f$ setpoint. These transitions are thus ascribed to the changes of tip apex. If the dot-like and rod-like features represented different structures of the monolayer accordingly, then transformations from one to the other would require a displacement of numerous molecules and take time to complete.

As will be described in Section 3.2, liquid water layers were present on the adsorbed mercaptohexanol monolayer to produce oscillatory modulations in the $\Delta f$-distance curves. On an oscillatory $\Delta f$-distance curve, the bistable tip-surface distance is allowed even though feedback is regulated with one $\Delta f$ setpoint. In fact, tip jumps from one stable distance to the other were experienced on interfaces with bistable curves [28, 29]. However, the transitions in topography found in Figure 2 cannot be ascribed to tip jumps, since the locally averaged topographic height continuously drifted without an abrupt shift assignable to a tip jump across a water layer.

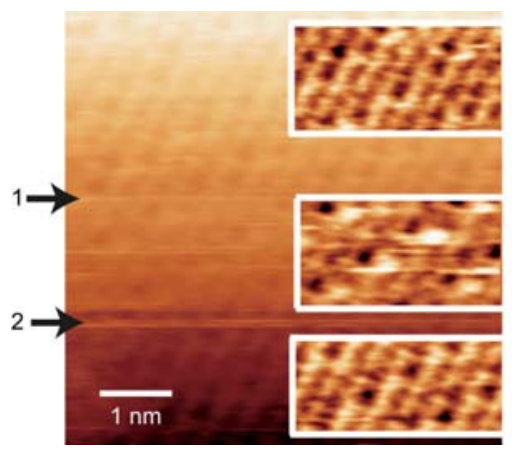

Fig. 2. Topography of a mercaptohexanol monolayer with reversible changes from the rod-like features to the dot-like feature. Two transitions occurred on the line scans marked with the numbered arrows. The cantilever oscillation amplitude was $0.1 \mathrm{~nm}$ and the frequency shift setpoint was $+300 \mathrm{~Hz}$. 
A topographic appearance that is sensitive to the tip apex has frequently been found in scanning probe studies [30]. As an example related to the current study, Ito et al. [31] found a single-row appearance of 1-octadecanol lamellae physisorbed on graphite. Their STM tip was modified with thiols that can form hydrogen bonds with a scanned object. The single row protruding from the surface was attributed to enhanced tunnel current on hydroxyl end-groups of octadecanol hydrogen-bonded to the thiols.

Consider a similar phenomenon with the AFM tip on the mercaptohexanol monolayer. It is natural in the aqueous imaging solution for the tip to pick up some molecular residue capable of hydrogen-bonding. The $\mathrm{OH}$ and $\mathrm{SH}$ end-groups, the latter of which may have dissociated on gold, can produce an enhanced tip-surface force, relative to the $n$-hexyl body of each molecule. In this scheme of interpretation, the dot-like protrusions represent the end-groups with the enhanced force. When the assumed hydrogen-bonding-capable residue is absent at the tip apex, the inert apex simply traces the physical topography of the adsorbed mercaptohexanol to generate the rod-like features. A similar transition of FM-AFM topography was observed on an $n$-decanol monolayer adsorbed on graphite [32], where bright protrusions appeared at the edges or centers of decanol lamellae corresponding with tip changes.

Figure 3 illustrates the assumed scheme of the topographic transition. A tip with or without the hydrogen-bonding-capable residue interacts with the end-groups and the body of the mercaptohexanol with different strengths. The symmetry of the topography determined in the constant tip-surface force regulation is sensitive to the composition of the apex. This scheme can generally be applied to a surface composed of chemically heterogeneous portions. When the surface is homogenous in chemical properties, the symmetry of the constant force topography is insensitive to tip changes. However, the absolute force strength is sensitive to tip changes.

Intentional tip modification for chemically selective FM-AFM has been successfully examined using an azobenzene derivative placed on tip apex by Takamatsu et al [33]. More quantitative relation of force and tip apex composition is an open issue for future studies. 
(a)

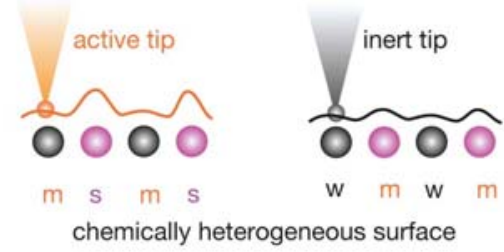

(b)

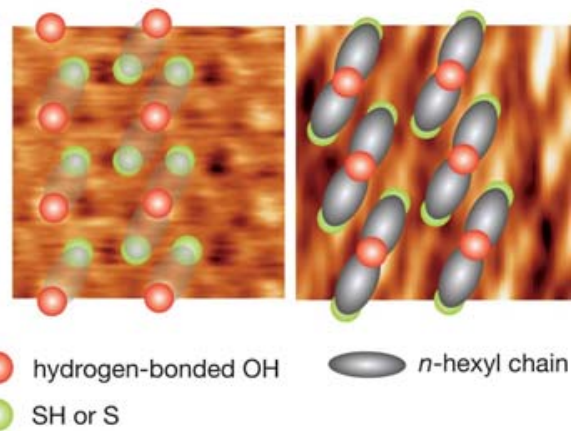

Fig. 3. Constant tip-surface force topography sensitive to tip apex composition. (a) A general schematic illustrating the sensitivity. s, $m$ and $\mathrm{w}$ represent strong, medium and weak tip-surface force. (b) The assignment of the different topographic appearances observed on the mercaptohexanol monolayer.

\subsection{Water structure over the monolayer}

A two-dimensional $\Delta f$ distribution was obtained on planes cross-sectional to the surface covered by the $(3 \times 2 \sqrt{3})$ monolayer. The tip was scanned vertically from the solution to the surface until $\Delta f$ exceeded a threshold $(+700 \mathrm{~Hz}$ in the present study) and then retracted into the solution to avoid contact with the surface. One $\Delta f$-distance curve was determined at one lateral coordinate. By repeating vertical scans at different lateral positions, a $\Delta f$ distribution was constructed on a plane cross-sectional to the surface with a desired azimuth. The typical acquisition time was $10 \mathrm{~s}$ per frame composed of 128 scan lines.

Figure 4 presents two $\Delta f$ distributions observed on the surface of the dot-like features. The azimuth of cross-sectional planes was set to be parallel to the long axis of the rectangular unit cell. A repulsive (or attractive) tip-surface force causes a positive (or negative) frequency shift. The relationship between the force and $\Delta f$ is nonlinear [34]. Here, the observed $\Delta f$ distribution is obtained as an approximate force distribution.

In the distribution of Figure 4(a), the brightest region appeared at the boundary of the solution and the solid. When the tip went down into that region, the repulsive force received by the tip monotonically increased. This is a sign of partial contact between the tip and the solid. Hence the envelope of the brightest region represents the surface topography. The surface 
topography traced by the bright envelope exhibited major and minor protrusions aligned periodically. The distance between neighboring major protrusions was $1.0 \mathrm{~nm}$, this is consistent with the unit cell length. A solid line was overlaid to trace the envelope. The trace reproduced the topographic cross-section independently obtained with the constant $\Delta f$ feedback regulation of tip-surface distance. The topographic cross-section (i) in Figure 4(c) was constructed on the line connecting the brightest dot-like protrusions. Major and minor protrusions appeared alternately.

In the solution side of the interface, a structured density was found as dark-to-bright changes in the $\Delta f$ distribution marked with dashed circles. The structured regions periodically appeared on top of the major protrusions on the surface. Following the assignment proposed in studies by the authors and other researchers [17-23], the modulated $\Delta f$ distribution is ascribed to localized interfacial water. The lateral location of localized water is consistent with the structure proposed in Figure 1(c), where $\mathrm{OH}$ end-groups are present at the brightest protrusions. Water localization on an $\mathrm{OH}$ end-group is reasonable. The sign of water structuring, that is, dark-to-bright changes in the $\Delta f$ distribution, was absent between the major protrusions. In the proposed structure, the gold substrate is exposed to the solution at the middle of the major protrusions, where specific interactions of the surface and water cannot be expected. Frequency-shift distribution was observed also on rod-like featured surfaces. It was unfortunately difficult to identify $\mathrm{OH}$ end-groups in the bright envelope at the bottom of the distribution. The two $\Delta \mathrm{f}$ distributions, one on the dot-like feature and the other on the rod-like feature, were not compared.

The distribution in Figure 4(b) was observed on a different plane from that of Figure 4(a). The topographic trace at the bottom contained twin protrusions with a trench between neighboring twins. This trace reproduced the topographic cross-section (ii) of Figure 4(c) and thus evidenced that the $\Delta f$ distribution was determined on a plane involving the line (ii). Modulated water density, marked by dashed circles, was present on each twin. In the proposed structure, the twinned protrusion is located at the pair of SH (or S) end-groups on the surface. The center of each twin is at the middle of the two $\mathrm{OH}$ end-groups. The local water density distribution enhanced on top of the $\mathrm{OH}$ end-groups should have a finite size in space. Hence the modulated water density captured in Figure 4(b) may be interpreted as the tail of the enhanced density distribution. The thiolate end-group on the surface, which can be polar, also possibly interacts with water to contribute to the modulated water density on the twin center. 
(a)

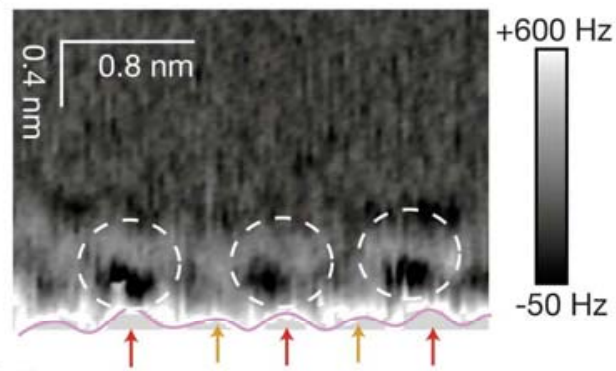

(c) (i)

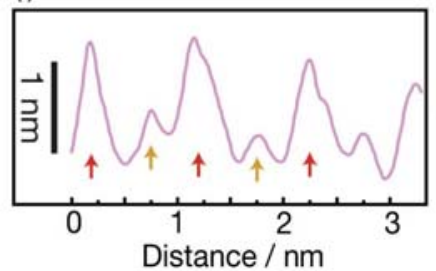

(b)

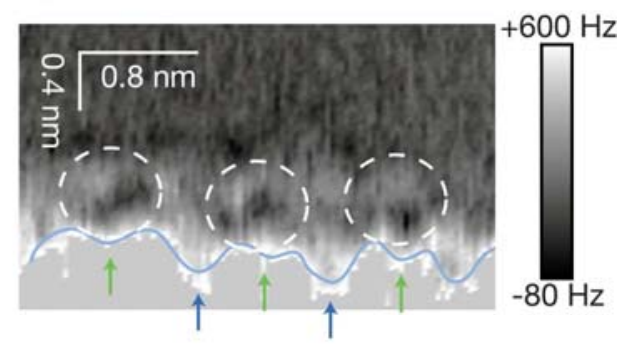

(ii)
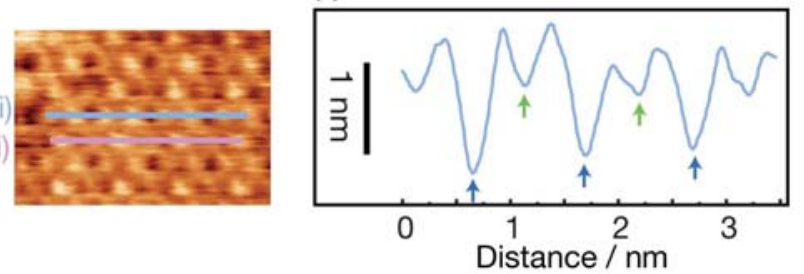

Fig. 4. $(\mathrm{a}, \mathrm{b})$ The $\Delta f$ distributions over the mercaptohexanol monolayer observed in different planes cross-sectional to the surface. The solid line in each distribution indicates the surface topography. Regions of modulated $\Delta f$ are marked with dashed circles. The cantilever oscillation amplitude is $0.1 \mathrm{~nm}$. (c) Cross-sectional height profiles constructed on line (i) and line (ii) in the constant $\Delta f$ topography shown in Figure 1(a). The arrows indicate periodic protrusions or trenches in the topography.

\section{Conclusions}

A mercaptohexanol monolayer assembled on gold (111) was probed in a $\mathrm{KCl}$ solution using frequency-modulation atomic force microscopy. Based on the constant $\Delta f$ topography of the surface and the cross-sectional $\Delta f$ distribution in the solution, a possible structure for the assembly was proposed: two mercaptohexanol molecules lying down in a rectangular $(3 \times 2 \sqrt{ } 3)$ unit cell. The dot-like and rod-like topographic features transformed each other due to tip changes. The modulated density of the solution was recognized on top of the topographic protrusions of the surface. This suggested liquid structuring induced by heterogeneous surface composition, i.e., water localization on the polar end-groups of the adsorbed mercaptohexanol.

Acknowledgment. The microscope used in this study was developed by the Advanced Measurement and Analysis Project of the Japan Science and Technology Agency in collaboration with Masahiro Ohta, Kazuyuki Watanabe, Noriaki Oyabu, Kei Kobayashi, Hirofumi Yamada, and Ryohei Kokawa. This work was supported by a Grant-in-Aid for Scientific Research on Priority Areas [477] and for Scientific Research (B) with Grant Numbers 
19056006 and 25286009. Takumi Hiasa was supported by the Japan Society for the Promotion of Science fellowship.

\section{References}

[1] G. E. Poirier, Characterization of organosulfur molecular monolayers on $\mathrm{Au}(111)$ using scanning tunneling microscopy, Chem. Rev. 97 (1997) 1117-1128.

[2] J. C. Love, L. A. Estroff, J. K. Kriebel, R. G. Nuzzo, G. M. Whitesides, Self-assembled monolayers of thiolates on metals as a form of nanotechnology, Chem. Rev. 105 (2005) 1103-1170.

[3] P. Fenter, P. Eisenberger, K. S. Liang, Chain-length dependence of the structures and phases of $\mathrm{CH}_{3}\left(\mathrm{CH}_{2}\right) n_{-1}$ SH self-assembled on Au(111), Phys. Rev. Lett. 70 (1993) 2447-2450.

[4] M. J. Giz, B. Duong, N. J. Tao, In situ STM study of self-assembled mercaptopropionic acid monolayers for electrochemical detection of dopamine, J. Electroanalytical Chem. 465 (1999) $72-79$.

[5] M. Petri, D. M. Kolb, U. Memmert, H. Meyer, Adsorption of mercaptopropionic acid onto $\mathrm{Au}(111)$ : Part I. Adlayer formation, structure and electrochemistry, Electrochimica Acta 49 (2003) 175-182.

[6] J. Zhang, Qijin, J. Ulstrup, Assembly dynamics and detailed structure of 1-propanethiol monolayers on $\mathrm{Au}(111)$ surfaces observed real time by in situ STM, Langmuir 22 (2006) 6203-6213.

[7] S. M. Mendoza, I. Arfaoui, S. Zanarini, F. Paolucci, P. Rudolf, Improvements in the characterization of the crystalline structure of acid-terminated alkanethiol self-assembled monolayers on $\mathrm{Au}(111)$, Langmuir 23 (2007) 582-588.

[8] C. Dubois, F. Stellacci, Self-assembled monolayer of short carboxyl-terminated molecules investigated with ex situ scanning tunneling microscopy, J. Phys. Chem. C 112 (2008) 7431-7435.

[9] G. E. Poirier, E. D. Pylant, J. M. White, Crystalline structures of pristine and hydrated mercaptohexanol self-assembled monolayers on $\mathrm{Au}(111)$, J. Chem. Phys. 105 (1996) 2089-2092.

[10] Y.-F. Liu, Y.-C. Yang, Y.-L. Lee, Assembly behavior and monolayer characteristics of OH-terminated alkanethiol on $\mathrm{Au}(111)$ : in situ scanning tunneling microscopy and electrochemical studies, Nanotechnology 19 (2008) 065609.

[11] T. Fukuma, M. Kimura, K. Kobayashi, K. Matsushige, H. Yamada, Development of low 
noise cantilever deflection sensor for multienvironment frequency-modulation atomic force microscopy, Rev. Sci. Instrum. 76 (2005) 053704.

[12] T. Fukuma, K. Kobayashi, K. Matsushige, H. Yamada, True atomic resolution in liquid by frequency-modulation atomic force microscopy, Appl. Phys. Lett. 87 (2005) 034101.

[13] S. Rode, N. Oyabu, K. Kobayashi, H. Yamada, A. Kühnle, True atomic-resolution imaging of (1014) calcite in aqueous solution by frequency modulation atomic force microscopy, Langmuir 25 (2009) 2850-2853.

[14] K. Nagashima, M. Abe, S. Morita, N. Oyabu, K. Kobayashi, H. Yamada, M. Ohta, R. Kokawa, R. Murai, H. Matsushima, H. Adachi, K. Takanao, S. Murakami, Molecular resolution investigation of tetragonal lysozyme (110) face in liquid by frequency-modulation atomic force microscopy, J. Vac. Sci. Technol. B 28 (2010) C4C11.

[15] M. J. Higgins, M. Polcik, T. Fukuma, J. E. Sader, Y. Nakayama, S. P. Jarvis, Structured water layers adjacent to biological membranes, Biophys. J. 91 (2006) 2532-2542.

[16] S. Ido, K. Kimura, N. Oyabu, K. Kobayashi, M. Tsukada, K. Matsushige, H. Yamada, Beyond the helix pitch: direct visualization of native DNA in aqueous solution, ACS Nano 7 (2013) 1817-1822.

[17] K. Kimura, S. Ido, N. Oyabu, K. Kobayashi, Y. Hirata, T. Imai, H. Yamada, Visualizing water molecule distribution by atomic force microscopy, J. Chem. Phys. 132 (2010) 194705.

[18] T. Fukuma, Y. Ueda, S. Yoshioka, H. Asakawa, Atomic-scale distribution of water molecules at the mica-water interface visualized by three-dimensional scanning force microscopy, Phys. Rev. Lett. 104 (2010) 016101.

[19] T. Hiasa, K. Kimura, H. Onishi, M. Ohta, K. Watanabe, R. Kokawa, N. Oyabu, K. Kobayashi, H. Yamada, Aqueous solution structure over $\alpha-\mathrm{Al}_{2} \mathrm{O}_{3}(01-12)$ probed by frequency-modulation atomic force microscopy, J. Phys. Chem. C 114 (2010) 21423-21426.

[20] T. Hiasa, K. Kimura, H. Onishi, Hydration of hydrophilic thiolate monolayers visualized by atomic force microscopy, Phys. Chem. Chem. Phys. 14 (2012) 8419-8424.

[21] H. Asakawa, S. Yoshioka, K. Nishimura, T. Fukuma, Spatial distribution of lipid headgroups and water molecules at membrane/water interfaces visualized by three-dimensional scanning force microscopy, ACS Nano 6 (2012) 9013-9020.

[22] T. Hiasa, K. Kimura, H. Onishi, Two-dimensional distribution of liquid hydrocarbons facing alkanethiol monolayers visualized by frequency modulation atomic force microscopy, Coll. Surf. A 396 (2012) 203-207.

[23] R. Nishioka, T. Hiasa, K. Kimura, H. Onishi, Specific hydration on p-nitroaniline crystal 
studied by atomic force microscopy, J. Phys. Chem. C 117 (2013) 2939-2943.

[24] J. P. Bucher, L. Santesson, K. Kern, Selective imaging of self-assembled monolayers by tunneling microscopy, Appl. Phys. A 59 (1994) 135-138.

[25] G. E. Poirier, M. J. Tarlov, The $\mathrm{c}(4 \times 2)$ superlattice of $n$-alkanethiol monolayers self-assembled on $\mathrm{Au}(111)$, Langmuir 10 (1994) 2853-2386.

[26] M. Yu, N. Bovet, C. J. Satterley, S. Bengió, K. R. J. Lovelock, P. K. Milligan, R. G. Jones, D. P. Woodruff, V. Dhanak, True nature of an archetypal self-assembly system: mobile Au-thiolate species on Au(111), Phys. Rev. Lett. 97 (2006) 166102.

[27] H. Sellers, A. Ulman, Y. Shnidman, J. E. Eilers, Structure and binding of alkanethiolates on gold and silver surfaces: implications for self-assembled monolayers, J. Am. Chem. Soc. 115 (1993) 9389-9401.

[28] T. Fukuma, M. J. Higgins, S. P. Jarvis, Direct imaging of individual intrinsic hydration layers on lipid bilayers at Ångstrom resolution, Biophys. J. 92 (2007) 3603-3609.

[29] S.-H. Loh, S. P. Jarvis, Visualization of ion distribution at the mica-electrolyte interface, Langmuir 26 (2010) 9176-9178.

[30] Y. Gan, Atomic and subnanometer resolution in ambient conditions by atomic force microscopy, Surf. Sci. Rep. 64 (2009) 99-121.

[31] T. Ito, P. Bühlmann, Y. Umezawa, Scanning tunneling microscopy using chemically modified tips, Anal. Chem. 70 (1998) 255-259.

[32] T. Hiasa, K. Kimura, H. Onishi, Cross-sectional structure of liquid 1-decanol over graphite, J. Phys. Chem. C 116 (2012) 26475-26479.

[33] D. Takamatsu, Y. Yamakoshi, K. Fukui, Photoswitching behavior of a novel single molecular tip for noncontact atomic force microscopy designed for chemical identification, $\mathrm{J}$. Phys. Chem. B 110 (2006) 1968-1970

[34] J. E. Sader, S. P. Jarvis, Accurate formulas for interaction force and energy in frequency modulation force spectroscopy, Appl. Phys. Lett. 84 (2004) 1801-1803. 